\title{
Scalar Nonstandard Interactions in Neutrino Oscillation
}

\author{
Shao-Feng Ge $\mathrm{Ge}^{1,2,3,4,5, *}$ and Stephen J. Parke ${ }^{5}$ \\ ${ }^{1}$ Kavli IPMU (WPI), UTIAS, University of Tokyo, Kashiwa, Chiba 277-8583, Japan \\ ${ }^{2}$ Department of Physics, University of California, Berkeley, California 94720, USA \\ ${ }^{3}$ T. D. Lee Institute, Shanghai 200240, China \\ ${ }^{4}$ School of Physics and Astronomy, Shanghai Jiao Tong University, Shanghai 200240, China \\ ${ }^{5}$ Theoretical Physics Department, Fermi National Accelerator Laboratory, Batavia, Illinois 60510, USA
}

(Received 10 January 2019; revised manuscript received 6 March 2019; published 29 May 2019)

\begin{abstract}
The scalar nonstandard interactions (NSI) can also introduce matter effect for neutrino oscillation in a medium. Especially the recent Borexino data prefer nonzero scalar NSI, $\eta_{e e}=-0.16$. In contrast to the conventional vector NSI, the scalar type contributes as a correction to the neutrino mass matrix rather than the matter potential. Consequently, the scalar matter effect is energy independent while the vector one scales linearly with neutrino energy. This leads to significantly different phenomenological consequences in reactor, solar, atmospheric, and accelerator neutrino oscillations. A synergy of different types of experiments, especially those with matter density variation, is necessary to identify the scalar NSI and guarantee the measurement of $C P$ violation at accelerator experiments.
\end{abstract}

DOI: 10.1103/PhysRevLett.122.211801

Introduction.-Neutrino oscillation was originally proposed to happen in a vacuum due to a nontrivial neutrino mass matrix [1]. The matter potential that neutrinos experience when propagating through matter medium was recognized by Wolfenstein in 1978 [2]. If the matter density times the neutrino energy is at the right value, mixing angles can resonant to the maximal value and significantly change the oscillation behavior [3]. The MSW effect $[2,3]$ successfully explains the observed solar neutrino fluxes [4] and makes a consistent picture with the terrestrial experiments [5]. Matter effects have played a very important role in our understanding of neutrino oscillations.

In the very first paper [2] on the neutrino matter effect, Wolfenstein introducted nonstandard interactions with generally parametrized vector and axial-vector currents. This opens up the possibility of using neutrino oscillation to probe extra new physics beyond the standard model (SM), in addition to the neutrino mass matrix [6]. The effect of NSI creates problems for the Dirac $C P$ phase measurement. Both real diagonal and complex off-diagonal elements can fake the $C P$ violation effect and hence disguise the genuine Dirac $C P$ phase [7].

Not only the ordinary matter can induce the matter effect, but also a dark sector medium such as dark energy [8], or

Published by the American Physical Society under the terms of the Creative Commons Attribution 4.0 International license. Further distribution of this work must maintain attribution to the author(s) and the published article's title, journal citation, and DOI. Funded by SCOAP ${ }^{3}$.
MaVaNs (mass varying neutrinos) [9], fuzzy dark matter (DM) [10], or with a stand-alone particle [11]. Although the dark sector density is much lower than the ordinary matter density in the Sun or Earth, a large enough effect is possible for superlight mediators. In the extreme environment of supernova, NSIs can affect the collective oscillation of neutrinos [12].

Coming back to the effect of NSI induced by ordinary matter on neutrino oscillations, the discussion so far has been focusing on vector currents, either from a vector mediator or with Fierz transformation from a charged scalar [13]. Neutrinos can couple to not only vector field, but also scalar field. To some extent, neutrino coupling with scalar field is even more natural than the vector one. Because of the observed oscillation, at least two of the three light neutrinos are massive. A natural mechanism for neutrinos to acquire masses is via coupling with a scalar that has nonzero vacuum expectation values. Such a possibility cannot be easily excluded. Since the left-handed neutrino belongs to $S U(2)_{L}$ doublets and hence the scalar coupling with neutrino may also couple with charged leptons, for example via mixing with the neutral Higgs boson, it is natural to see the matter effect induced by such a scalar particle.

We point out in this Letter that the scalar NSI can introduce rich phenomenology in reactor, solar, atmospheric, and accelerator experiments. It is inevitable to use a synergy of multiple experiments to test the scalar NSI and guarantee the $C P$ measurement against the scalar NSI. Although the matter effect can also arise from the scalar mediator in MaVaNs [14] and fuzzy dark matter [10] scenarios, its size is not proportional to the ordinary matter 
density. Either a matter effect is modulated by dark energy, neutrino, or dark matter densities, or is proportional to a nontrivial function of the matter density such as $\tanh \rho$. In contrast, we make model-independent study of the scalar NSI that scales with constant proportionality to the ordinary matter density and has rich phenomenological consequences.

Matter potential and NSI.-Before exploring the scalar NSI, let us first take a look how the vector interactions can introduce matter potential in neutrino oscillation [15]. In the SM, the matter potential can be induced by both charged and neutral currents. With Fierz transformation [16], the effective Lagrangian contributed by charged current $\propto\left[\bar{e}\left(p_{1}\right) \gamma^{\mu} P_{L} \nu_{e}\left(p_{2}\right)\right]\left[\overline{\nu_{e}}\left(p_{3}\right) \gamma^{\nu} P_{L} e\left(p_{4}\right)\right]$ turns into the neutral current form [17]

$$
\mathcal{L}_{\mathrm{cc}}^{\mathrm{eff}}=-\frac{4 G_{F}}{\sqrt{2}}\left[\overline{\nu_{e}}\left(p_{3}\right) \gamma_{\mu} P_{L} \nu_{e}\left(p_{2}\right)\right]\left[\bar{e}\left(p_{1}\right) \gamma^{\mu} P_{L} e\left(p_{4}\right)\right]
$$

Here we use electrons as the environmental matter for illustration and the same procedure applies for protons and neutrons. The matter effect comes from the so-called forward scattering that experiences zero momentum transfer, $p_{1}=p_{4} \equiv p_{e}$ and $p_{2}=p_{3} \equiv p_{\nu}$, and hence is momentum independent. We can obtain the matter potential by sandwiching the effective Lagrangian with in and out states,

$$
\begin{aligned}
& V_{c c}=-\left\langle\nu_{e} e\left(p_{e}, s_{e}\right)\left|\mathcal{L}_{\mathrm{eff}}^{\mathrm{cc}}\right| \nu_{e} e\left(p_{e}, s_{e}\right)\right\rangle, \\
& \bar{V}_{c c}=-\left\langle\bar{\nu}_{e} e\left(p_{e}, s_{e}\right)\left|\mathcal{L}_{\mathrm{eff}}^{\mathrm{cc}}\right| \bar{\nu}_{e} e\left(p_{e}, s_{e}\right)\right\rangle,
\end{aligned}
$$

for the neutrino and antineutrino cases, respectively. With the neutrino and electron spinors separated, we can first focus on the neutrino parts, $\left\langle\nu_{e}\left|\bar{\nu}_{e} \gamma_{\mu} P_{L} \nu_{e}\right| \nu_{e}\right\rangle$ and $\left\langle\bar{\nu}_{e}\left|\bar{\nu}_{e} \gamma_{\mu} P_{L} \nu_{e}\right| \bar{\nu}_{e}\right\rangle$. The quantized neutrino spinor is $\nu_{e} \equiv$ $a u+b^{\dagger} v$ where $a$ and $b$ are the annihilation operators of neutrino and antineutrino, respectively. Correspondingly, the external states are $\left|\nu_{e}\right\rangle \equiv a^{\dagger}|0\rangle$ and $\left|\bar{\nu}_{e}\right\rangle \equiv b^{\dagger}|0\rangle$. The anticommunication property of the neutrino operators leads to opposite sign between the neutrino and antineutrino matter potentials $V_{\mathrm{cc}}=-\bar{V}_{\mathrm{cc}}=\sqrt{2} G_{F} n_{e}$.

For the vector NSI, the only difference is introducing an extra vector mediator between various neutrino flavors. In all these cases, the matter effect contributes to the $\bar{\nu} \gamma^{0} \nu$ term,

$$
\bar{\nu}_{\beta}\left[\left(i \partial_{t} \mp V_{\alpha \beta}^{(\dagger)}\right) \gamma^{0}-i \nabla \cdot \gamma-M_{\beta \alpha}\right] \nu_{\alpha}=0,
$$

with $-V_{\alpha \beta}$ for neutrino and $V_{\alpha \beta}^{\dagger}$ for antineutrino. When expanded to the linear order, the Hamiltonian for neutrino oscillation is

$$
\mathcal{H} \approx E_{\nu}+\frac{M M^{\dagger}}{2 E_{\nu}} \pm\left(V_{\mathrm{SI}}+V_{\mathrm{NSI}}\right)
$$

where the neutrino mass matrix $M$, the matter potential $V_{\mathrm{SI}}$ for the standard interaction (SI), and the NSI counterpart $V_{\text {NSI }}$ are $3 \times 3$ matrices. For simplicity, we denote $\left|p_{\nu}\right| \approx E_{\nu}$ since neutrino masses are tiny. Note that the neutrinos (antineutrinos) oscillation is described by $\mathcal{H}$ $\left(\mathcal{H}^{*}\right)$, respectively. The matter effect appears as an extra term added to the Hamiltonian or energy. Both the SI and NSI act as matter potential. For the matter potential to be important, either the neutrino energy or the matter density should be large enough, $2 E_{\nu} V \gtrsim \Delta m_{i j}^{2}$, where the latter has two typical values, $\Delta m_{21}^{2}=7.5 \times 10^{-5}$ or $\Delta m_{31}^{2}=2.7 \times 10^{-3} \mathrm{eV}^{2}$.

The scalar NSI.-The scalar NSI effect is no longer a matter potential. This is because the effective Lagrangian (1) is no longer composed of vector current but a scalar Yukawa term for Dirac neutrinos

$$
\mathcal{L}_{\text {eff }}^{s} \propto y_{f} Y_{\alpha \beta}\left[\bar{\nu}_{\alpha}\left(p_{3}\right) \nu_{\beta}\left(p_{2}\right)\right]\left[\bar{f}\left(p_{1}\right) f\left(p_{4}\right)\right],
$$

which cannot convert to vector currents [18]. In the nonrelativistic limit the environmental fermion spinor reduces to $u_{f}=(\xi, \xi)^{T}$, where $\xi_{+}=(1,0)^{T}$ or $\xi_{-}=$ $(0,1)^{T}$ for the two spin polarizations. Consequently, $\bar{u}_{f} u_{f}=\bar{u}_{f} \gamma^{0} u_{f}=2 \xi^{\dagger} \xi=n_{f}$, reducing to the matter density. The correction to the Dirac equation (3) then shifts to the mass term

$$
\bar{\nu}_{\beta}\left[i \partial_{\mu} \gamma^{\mu}+\left(M_{\beta \alpha}+\frac{\sum_{f} n_{f} y_{f} Y_{\alpha \beta}}{m_{\phi}^{2}}\right)\right] \nu_{\alpha}=0,
$$

where $y_{f}$ is the Yukawa coupling of the scalar mediator $\phi$ with the environmental fermion $f, Y_{\alpha \beta}$ is the one with neutrinos, $Y_{\alpha \beta} \bar{\nu}_{\alpha} \phi \nu_{\beta}+$ H.c., and for a real scalar $Y=Y^{\dagger}$. Defining $\delta M \equiv \sum_{f} n_{f} y_{f} Y / m_{\phi}^{2}$, the effective Hamiltonian (4) becomes

$$
\mathcal{H} \approx E_{\nu}+\frac{(M+\delta M)(M+\delta M)^{\dagger}}{2 E_{\nu}} \pm V_{\mathrm{SI}}
$$

The scalar NSI appears as a correction to the neutrino mass term, rather than the matter potential. Since the matter effect is inversely proportional to the mediator mass, $1 / \mathrm{m}_{\phi}^{2}$, large enough $\delta M$ is possible with a light enough scalar that can survive current constraints from supernova [19], meson and lepton decays [20], neutrino electron scattering [21], neutrino trident production [22], Big Bang Nucleosynthesis [23], coherent scattering [24], stellar cooling [25], and other various observables [27]. None of them can exclude the parameter region with both the mediator mass and couplings being tiny.

The neutrino mass matrix $M=V_{\nu} D_{\nu} V_{\nu}^{\dagger}$ can be diagonalized by the mixing matrix $V_{\nu} \equiv P_{\nu} U_{\nu} Q_{\nu}$, where $P_{\nu}$ and $Q_{\nu}$ are diagonal rephasing matrices, $U_{\nu}$ the PMNS matrix, and $D_{\nu} \equiv \operatorname{diag}\left\{m_{1}, m_{2}, m_{3}\right\}$ is the diagonal mass matrix. 
With $\delta M$, the unphysical rephasing matrix $P_{\nu}$ cannot be rotated away. To comply with the established conventions, we rotate $P_{\nu}$ into the scalar NSI contribution, $M \rightarrow \widetilde{M} \equiv$ $U_{\nu} D_{\nu} U_{\nu}^{\dagger}$ and $\delta M \rightarrow \delta \widetilde{M} \equiv P_{\nu}^{\dagger} \delta M P_{\nu}$. For easy comparison with the genuine mass term, we parametrize the scalar NSI as

$$
\delta \widetilde{M} \equiv \sqrt{\Delta m_{31}^{2}}\left(\begin{array}{lll}
\eta_{e e} & \eta_{e \mu} & \eta_{e \tau} \\
\eta_{\mu e} & \eta_{\mu \mu} & \eta_{\mu \tau} \\
\eta_{\tau e} & \eta_{\tau \mu} & \eta_{\tau \tau}
\end{array}\right),
$$

where $\eta_{\alpha \beta}$ are dimensionless parameters. Note that those phases in $\delta \widetilde{M}$ are combinations of the unphysical phases from $P_{\nu}$ and the scalar NSI matrix $\delta M$. In the presence of the scalar NSI, the unphysical phases in $P_{\nu}$ can also have physical consequences on neutrino oscillation. The same thing happens to the absolute mass scale that can be subtracted from $M M^{\dagger}$ as a common $m_{i}^{2}$ term but is always associated with the scalar NSI contribution as $M \delta M^{\dagger}+M^{\dagger} \delta M$. In this sense, the scalar NSI is also totally different from the vector NSI.

The vector NSI always conserves chirality which is no longer true for the scalar NSI. The latter can only appear as a correction to the neutrino mass term that flips chirality [28,29], contrary to previous studies [30]. Note that the scalar NSI is not suppressed by either the nonrelativistic environmental fermion $f,\left\langle m_{f} / E_{f}\right\rangle \approx 1$ [28], or the relativistic neutrinos since neutrino oscillation directly probes the neutrino mass term, $M+\delta M$. As long as the correction $\delta M$ is comparable with the already tiny neutrino mass term $M$, the scalar NSI can have a sizable effect.

Phenomenological consequences.-Neutrino oscillation probes not only neutrino mixing but also the neutrino interactions with the medium. Wolfenstein pointed out that "even if all neutrinos are massless it is possible to have oscillations occur when neutrinos pass through matter" [2]. He estimated "the oscillation length in matter of normal density is of the order $10^{9} \mathrm{~cm}$ " $\left(10^{4} \mathrm{~km}\right)$, which is inversely proportional to the matter potential. In the absence of the genuine mass term, the vector NSI leads to an oscillation phase $e^{i V L}$ and hence the oscillation length $L \propto 1 / V$ is independent of the neutrino energy but is only a function of the medium density.

Nowadays, we have already measured neutrino oscillation attributed to mass splittings that lead to an oscillation phase $e^{i \Delta m_{i j}^{2} L / 4 E_{\nu}}$ and hence the oscillation length is proportional to the neutrino energy, $L \propto E_{\nu} / \Delta m_{i j}^{2}$. But the question is whether the measured mass splittings are the genuine one $M$ from the fundamental Lagrangian or the faked mass matrix $\delta M$ by the scalar NSI. Even in the absence of the genuine mass matrix, oscillation can still happen due to the scalar NSI [31]. There is no essential difference between the genuine mass term and the one induced by the scalar NSI.

Its dependence on the matter density can help us to identify the scalar NSI. While the genuine mass matrix $M$ is independent of environmental conditions, the scalar NSI contribution $\delta \widetilde{M}$ scales with the matter density. The oscillation probability can feel the matter density variations along the baseline. For short-baseline terrestrial experiments, the variation in the matter density is negligible and one combination of $M$ and $\delta M$ can be redefined as the effectively measured mass matrix $\widetilde{M}_{\text {re }}$. Since the rector experiments such as KamLAND [32], Daya Bay [33], and RENO [34] give the most precise measurements, not to say the future JUNO [35], we implement the matter density subtraction at their typical matter density $\rho_{s}=$ $2.6 \mathrm{~g} / \mathrm{cm}^{3}$ [36],

$$
\widetilde{M}+\delta \widetilde{M}(\rho) \equiv \widetilde{M}_{\mathrm{re}}+\delta \widetilde{M}\left(\rho_{s}\right) \frac{\rho-\rho_{s}}{\rho_{s}},
$$

for both the neutrino and antineutrino modes. With $\rho=\rho_{s}$, the effective mass matrix is exactly the reconstructed one $\widetilde{M}_{\mathrm{re}} \equiv \widetilde{M}+\delta \widetilde{M}\left(\rho_{s}\right)=U_{\nu} D_{\nu} U_{\nu}^{\dagger}$, where $\delta \widetilde{M}\left(\rho_{s}\right)$ is actually Eq. (8) fixed at the subtraction density $\rho_{s}$. We implement these in the NuPro package [37] for the simulations across this Letter. Figure 1 shows that the scalar NSI has large effects at both Daya Bay and JUNO. Nevertheless, the reactor experiments are not enough since the density subtraction in Eq. (9) can eliminate the effects of the scalar NSI. A global effort of combining different types of experiments is necessary to distinguish the genuine neutrino mass matrix and the scalar NSI correction.

We show the effect of the scalar NSI on the solar neutrino convertion in Fig. 2, with a similar plot of the vector NSI for comparison. Different from the vector one, the scalar NSI is energy independent and hence not suppressed at low energy. In addition, the SNO neutrinos experience much higher matter density [38] than the KamLAND reactor neutrinos and hence can experience a sizable second term in Eq. (9) with $\rho \gg \rho_{s}$. Both mixing angles and mass squared differences are modified. This provides the possibility of explaining the discrepancy between the KamLAND [32] and SNO [39] measurements of the neutrino mass splitting $\Delta m_{21}^{2}$. The blue $\left(\eta_{\mu \mu}=-0.05\right)$ and green $\left(\eta_{\tau \tau}=-0.05\right)$ curves for the scalar NSI can mimic the black dashed curve for the SI with the best fit value $\Delta m_{21}^{2}=4.7 \times 10^{-5} \mathrm{eV}^{2} \quad$ [40]. Last year, the Borexino experiment made the first simultaneous
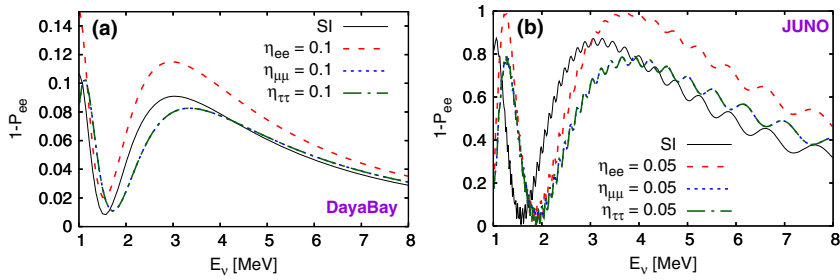

FIG. 1. The effect of the scalar NSI on the reactor antineutrino oscillation probabilities at (a) Daya Bay and (b) JUNO. 

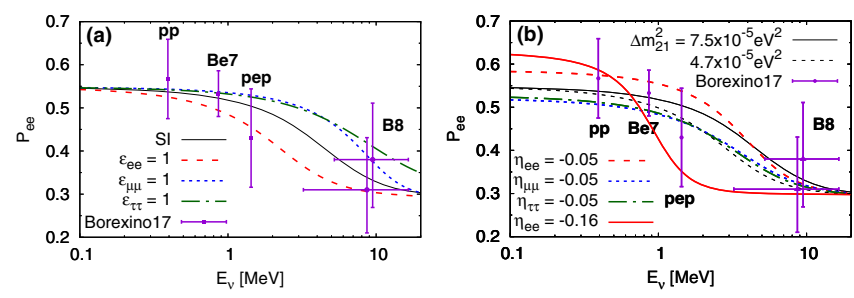

FIG. 2. The solar neutrino conversion probabilities with (a) the vector and (b) the scalar NSIs, together with the Borexino measurement [41] of the $p p,{ }^{7} \mathrm{Be}$, and pep fluxes.

measurement of the $p p,{ }^{7} \mathrm{Be}$, and $p e p$ fluxes [41]. As a naive estimation, we plot the $\chi^{2}$ function for these three data points in Fig. 3. The $\eta_{\mu \mu}$ and $\eta_{\tau \tau}$ elements are effectively the same for the solar neutrino oscillation probability and their best fit is the SM scenario. But for the $\eta_{e e}$ element, there is a local minimal around $\eta_{e e}=-0.16$ beyond which the $\chi^{2}$ curve has a sharp increase due to the flip of mass eigenvalues. The latest Borexino data [41] do favor a nonzero scalar NSI. We show the oscillation probability curve with $\eta_{e e}=-0.16$ in Fig. 2 for comparison. The preferred nonzero $\eta_{e e}$ is mainly fixed by the smaller central value of the pep flux than the SI prediction. Although the curve shape is quite different from the standard case, it is still consistent with the Borexino data, including the ${ }^{8} B$ flux from the earlier measurement [42]. The future $\mathrm{SNO}+$ [43] and Jinping [44] neutrino experiments are more precise and can help to pin it down.

The atmospheric neutrino oscillation can also experience matter density variation and hence help identify the scalar NSI. In Fig. 4 we show the atmospheric neutrino oscillogram and its modification by the vector or scalar NSI. Those neutrinos crossing the Earth core experience the most significant matter density variation. Consequently, the core region $\left(\cos \theta_{z} \lesssim-0.8\right)$ shows the largest effect where the difference in $P_{\mu e}$ is as large as 0.14 between the SI and the scalar NSI. Note that the maximal value of $P_{\mu e}$ is around 0.5 which is clearly seen in the decomposition formalism [45]. The relative change is as large as 1 in the energy range $E_{\nu} \lesssim 5 \mathrm{GeV}$. We expect the PINGU [46],

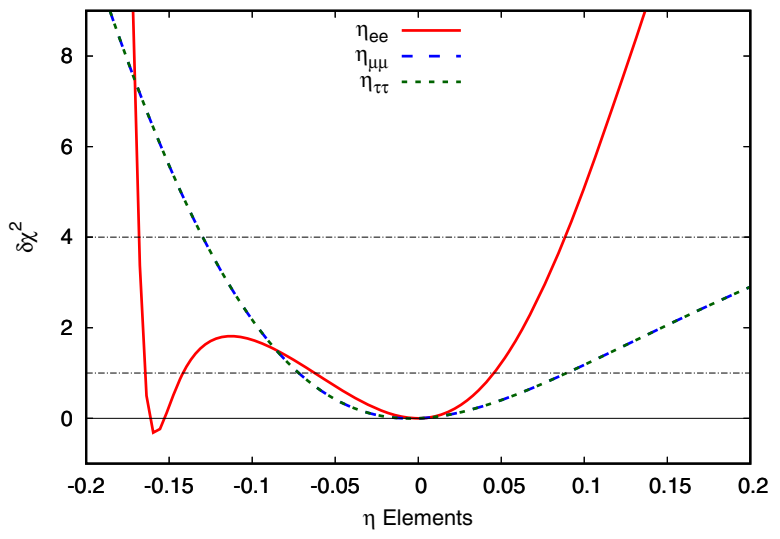

FIG. 3. The $\chi^{2}$ fit of the scalar NSI to the Borexino data [41].
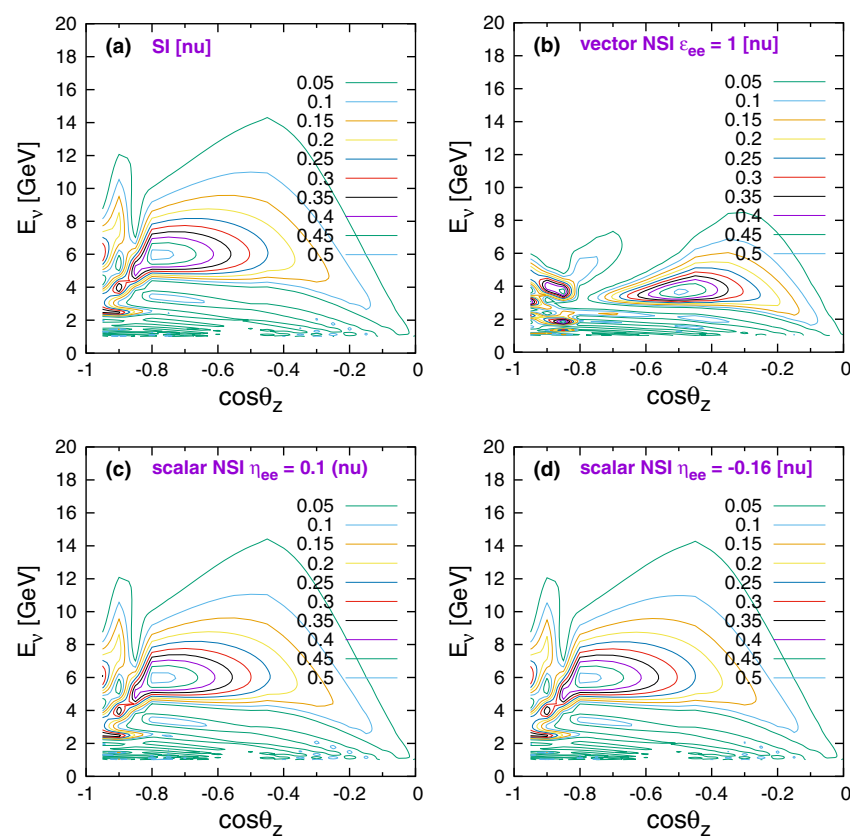

FIG. 4. The atmospheric neutrino oscillogram of $P_{\mu e}$ with (a) the SI only, (b) the vector NSI $\epsilon_{e e}=1$, and the scalar NSI either (c) $\eta_{e e}=0.1$ or (d) $\eta_{e e}=-0.16$.

ORCA [47], and INO [48] experiments to put some constraints on the scalar NSI. In addition, the lower energy threshold with Super-PINGU [49] can further enhance the sensitivity to the scalar NSI whose effect can surpass the matter potential at lower energy.

For the accelerator neutrino experiments, whose main purpose is for the Dirac $C P$ phase measurement, the situation is a little more intricate. Since the effective mass matrix is modified by the scalar NSI, the effective Dirac $C P$ phase can be quite different from the genuine one. We show the oscillation probability $P_{\mu e}$ at TNT2K [50], including the neutrino mode $\nu \mathrm{T} 2 \mathrm{~K}$ and the antineutrino mode $\mu \mathrm{SK}$, and both modes at DUNE [51] in Fig. 5. To make the faked $C P$ effect explicit, we remove the matter density
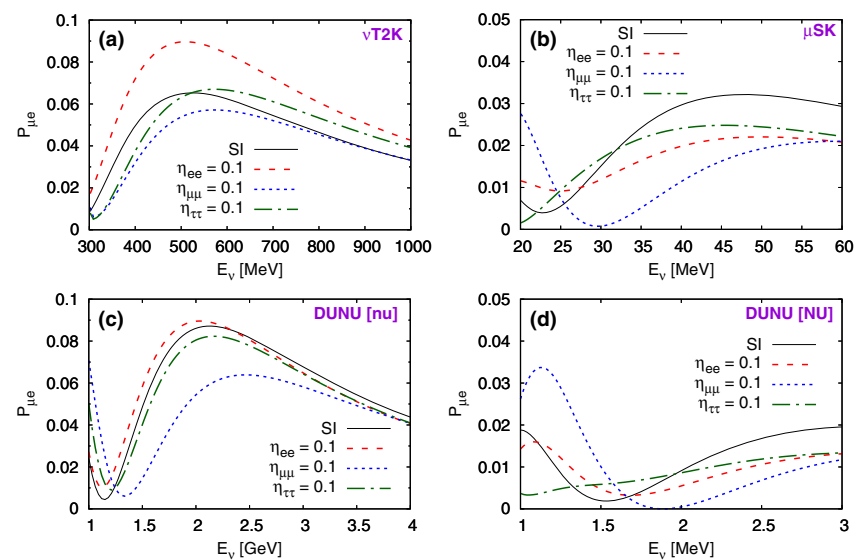

FIG. 5. The probability $P_{\mu e}$ at (a) $\nu \mathrm{T} 2 \mathrm{~K}$, (b) $\mu \mathrm{SK}$, as well as (c) neutrino and (d) antineutrino modes at DUNE. 
subtraction so that the effect of the scalar NSI appears in both neutrino and antineutrino modes. Although the neutrino energy varies a lot, all experiments receive comparable modification from the scalar NSI which is totally different from the vector case. If the complex phases and off-diagonal elements $\eta_{\alpha \beta}$ with $\alpha \neq \beta$ are also introduced, the situation would become even more complicated. The degeneracy between the genuine Dirac $C P$ phase and the scalar NSI can make the $C P$ measurement more difficult, in the same way as the vector NSI [7] and the nonunitary mixing [52].

Summary and discussions. - We point out that the scalar NSI has totally different features and phenomenological consequences from the vector one. The scalar NSI contributes as correction to the neutrino mass matrix and hence its effect is independent of the neutrino energy. Even for the low energy reactor, solar, and muon decay at rest ( $\mu \mathrm{DAR})$ experiments, the effect of the scalar NSI cannot be ignored. In addition, the scalar NSI can fake the $C P$ effect and becomes a trouble to the on-going and future $C P$ measurements at T2(H)K [53], NO $\nu \mathrm{A}$ [54], and DUNE [51]. A global effort of using matter-density-varying oscillation, such as the solar and atmospheric neutrino experiments, is then needed for precision measurement of the leptonic Dirac $C P$ phase.

We would like to thank Peter Denton, Jiajun Liao, and Alexei Smirnov for useful discussions and references after reading an earlier version of this manuscript. The work of S.F.G. was supported by JSPS KAKENHI Grant No. JP18K13536, World Premier International (WPI) Research Center Initiative, MEXT, Japan, and Fermilab Neutrino Physics Center Fellowship Award Program. S.F.G. is also grateful to the hospitality of Patrick Huber and the Center for Neutrino Physics at Virginia Tech where this Letter was partially drafted. This Letter was authored (S. J. P.) by Fermi Research Alliance, LLC under Contract No. DE-AC02-07CH11359 with the U.S. Department of Energy, Office of Science, Office of High Energy Physics. This project (S. J. P.) has received funding and support from the European Union's Horizon 2020 research and innovation programme under the Marie Sklodowska-Curie Grants Agreement No. 690575 and No. 674896.

*Corresponding author. gesf02@gmail.com

[1] B. Pontecorvo, Mesonium and anti-mesonium, Zh. Eksp. Teor. Fiz. 33, 549 (1957) [Sov. Phys. JETP 6, 429 (1957)]; Z. Maki, M. Nakagawa, and S. Sakata, Remarks on the unified model of elementary particles, Prog. Theor. Phys. 28, 870 (1962).

[2] L. Wolfenstein, Neutrino oscillations in matter, Phys. Rev. D 17, 2369 (1978).
[3] S. P. Mikheyev and A. Y. Smirnov, Resonance amplification of oscillations in matter and spectroscopy of solar neutrinos, Yad. Fiz. 42, 1441 (1985) [Sov. J. Nucl. Phys. 42, 913 (1985)]; S. P. Mikheev and A. Y. Smirnov, Resonant amplification of $\nu$ oscillations in matter and solar neutrino spectroscopy, Nuovo Cimento C 9, 17 (1986); S. P. Mikheev and A. Y. Smirnov, Neutrino oscillations in a variable density medium and neutrino bursts due to the gravitational collapse of stars, Sov. Phys. JETP 64, 4 (1986).

[4] J. N. Bahcall, Solar Neutrinos. I: Theoretical, Phys. Rev. Lett. 12, 300 (1964); R. Davis, Solar Neutrinos. II: Experimental, Phys. Rev. Lett. 12, 303 (1964); Q. R. Ahmad et al. (SNO Collaboration), Direct Evidence for Neutrino Flavor Transformation from Neutral Current Interactions in the Sudbury Neutrino Observatory, Phys. Rev. Lett. 89, 011301 (2002).

[5] P. F. de Salas, D. V. Forero, C. A. Ternes, M. Tortola, and J. W. F. Valle, Status of neutrino oscillations 2018: $3 \sigma$ hint for normal mass ordering and improved $C P$ sensitivity, Phys. Lett. B 782, 633 (2018).

[6] O. G. Miranda and H. Nunokawa, Non standard neutrino interactions: current status and future prospects, New J. Phys. 17, 095002 (2015); Y. Farzan and M. Tortola, Neutrino oscillations and non-standard interactions, Front. Phys. 6, 10 (2018).

[7] S. F. Ge and A. Y. Smirnov, Non-standard interactions and the $C P$ phase measurements in neutrino oscillations at low energies, J. High Energy Phys. 10 (2016) 138.

[8] P. Q. Hung, Sterile neutrino and accelerating universe, arXiv:hep-ph/0010126.

[9] R. Fardon, A. E. Nelson, and N. Weiner, Dark energy from mass varying neutrinos, J. Cosmol. Astropart. Phys. 10 (2004) 005; D. B. Kaplan, A. E. Nelson, and N. Weiner, Neutrino Oscillations as a Probe of Dark Energy, Phys. Rev. Lett. 93, 091801 (2004).

[10] A. Berlin, Neutrino Oscillations as a Probe of Light Scalar Dark Matter, Phys. Rev. Lett. 117, 231801 (2016); G. Krnjaic, P. A. N. Machado, and L. Necib, Distorted neutrino oscillations from time varying cosmic fields, Phys. Rev. D 97, 075017 (2018); V. Brdar, J. Kopp, J. Liu, P. Prass, and X. P. Wang, Fuzzy dark matter and nonstandard neutrino interactions, Phys. Rev. D 97, 043001 (2018); H. Davoudiasl, G. Mohlabeng, and M. Sullivan, Galactic dark matter population as the source of neutrino masses, Phys. Rev. D 98, 021301(R) (2018); J. Liao, D. Marfatia, and K. Whisnant, Light scalar dark matter at neutrino oscillation experiments, J. High Energy Phys. 04 (2018) 136; F. Capozzi, I. M. Shoemaker, and L. Vecchi, Neutrino oscillations in dark backgrounds, J. Cosmol. Astropart. Phys. 07 (2018) 004; G. y. Huang and N. Nath, Neutrinophilic axionlike dark matter, Eur. Phys. J. C 78, 922 (2018).

[11] G. J. Stephenson, Jr., J. T. Goldman, and B. H. J. McKellar, MSW - like enhancements without matter, Mod. Phys. Lett. A 12, 2391 (1997).

[12] Y. Yang and J. P. Kneller, Neutrino flavor transformation in supernovae as a probe for nonstandard neutrino-scalar interactions, Phys. Rev. D 97, 103018 (2018); J. F. Nieves and S. Sahu, Neutrino effective potential in a fermion and scalar background, Phys. Rev. D 98, 063003 (2018). 
[13] W. Chao and H. Zhang, One-loop renormalization group equations of the neutrino mass matrix in the triplet seesaw model, Phys. Rev. D 75, 033003 (2007); A. Abada, C. Biggio, F. Bonnet, M. B. Gavela, and T. Hambye, Low energy effects of neutrino masses, J. High Energy Phys. 12 (2007) 061; M. B. Gavela, D. Hernandez, T. Ota, and W. Winter, Large gauge invariant non-standard neutrino interactions, Phys. Rev. D 79, 013007 (2009); M. Malinsky, T. Ohlsson, and H. Zhang, Non-standard neutrino interactions from a triplet seesaw model, Phys. Rev. D 79, 011301(R) (2009).

[14] V. Barger, P. Huber, and D. Marfatia, Solar Mass-Varying Neutrino Oscillations, Phys. Rev. Lett. 95, 211802 (2005); M. Cirelli, M. C. Gonzalez-Garcia, and C. Pena-Garay, Mass varying neutrinos in the sun, Nucl. Phys. B719, 219 (2005); M. Blennow, T. Ohlsson, and W. Winter, Non-standard Hamiltonian effects on neutrino oscillations, Eur. Phys. J. C 49, 1023 (2007); V. Barger, D. Marfatia, and K. Whisnant, Confronting mass-varying neutrinos with MiniBooNE, Phys. Rev. D 73, 013005 (2006); M. C. Gonzalez-Garcia, P. C. de Holanda, and R. Zukanovich Funchal, Effects of environment dependence of neutrino mass versus solar and reactor neutrino data, Phys. Rev. D 73, 033008 (2006); T. Schwetz and W. Winter, Testing mass-varying neutrinos with reactor experiments, Phys. Lett. B 633, 557 (2006); P. H. Gu, X. J. Bi, B. Feng, B. L. Young, and X. Zhang, Detecting dark energy in long baseline neutrino oscillations, Chin. Phys. C 32, 530 (2008); P.C. de Holanda, Possible scenario for MaVaN's as the only neutrino flavor conversion mechanism in the Sun, J. Cosmol. Astropart. Phys. 07 (2009) 024; D. R. Gratieri and O.L.G. Peres, Searching for a nondiagonal mass varying mechanism in the $\nu_{\mu}-\nu_{\tau}$ system, Phys. Rev. D 90 , 013011 (2014).

[15] P. Langacker, J.P. Leveille, and J. Sheiman, On the detection of cosmological neutrinos by coherent scattering, Phys. Rev. D 27, 1228 (1983); H. A. Bethe, A Possible Explanation of the Solar Neutrino Puzzle, Phys. Rev. Lett. 56, 1305 (1986); J. Linder, Derivation of neutrino matter potentials induced by earth, arXiv:hep-ph/0504264.

[16] M. Fierz, Zur Fermischen Theorie des $\beta$-Zerfalls, Z. Phys. 104, 553 (1937).

[17] R. N. Mohapatra and P. B. Pal, Massive Neutrinos in Physics and Astrophysics, World Scientific Lecture Notes in Physics Vol. 60 (World Scientific, Singapore, New Jersey, London, Hong Kong, 1998), 2nd ed., p. 1; World Scientific Lecture Notes in Physics Vol. 72 (World Scientific, Singapore, New Jersey, London, Hong Kong, 2004), p. 1.

[18] J. F. Nieves and P. B. Pal, Generalized Fierz identities, Am. J. Phys. 72, 1100 (2004); C. C. Nishi, Simple derivation of general Fierz-like identities, Am. J. Phys. 73, 1160 (2005).

[19] L. Heurtier and Y. Zhang, Supernova constraints on massive (Pseudo)scalar coupling to neutrinos, J. Cosmol. Astropart. Phys. 02 (2017) 042.

[20] P. S. Pasquini and O. L. G. Peres, Bounds on neutrino-scalar Yukawa coupling, Phys. Rev. D 93, 053007 (2016); Erratum, Phys. Rev. D 93, 079902(E) (2016).

[21] M. Deniz, B. Sevda, S. Kerman, L. Singh, H. T. Wong, and M. Zeyrek, Constraints on scalar-pseudoscalar and tensorial non-standard interaction and tensorial unparticle couplings from neutrino-electron scattering, Phys. Rev. D 95, 033008 (2017).

[22] D. Geiregat et al. (CHARM-II Collaboration), First observation of neutrino trident production, Phys. Lett. B 245, 271 (1990); S. R. Mishra et al. (CCFR Collaboration), Neutrino Tridents and W Z Interference, Phys. Rev. Lett. 66, 3117 (1991); T. Adams et al. (NuTeV Collaboration), Neutrino trident production from NuTeV, High Energy Phys. Nucl. Phys. 1, 631 (1998); S. F. Ge, M. Lindner, and W. Rodejohann, Atmospheric trident production for probing new physics, Phys. Lett. B 772, 164 (2017).

[23] G. Y. Huang, T. Ohlsson, and S. Zhou, Observational constraints on secret neutrino interactions from big bang nucleosynthesis, Phys. Rev. D 97, 075009 (2018).

[24] Y. Farzan, M. Lindner, W. Rodejohann, and X. J. Xu, Probing neutrino coupling to a light scalar with coherent neutrino scattering, J. High Energy Phys. 05 (2018) 066.

[25] E. Hardy and R. Lasenby, Stellar cooling bounds on new light particles: plasma mixing effects, J. High Energy Phys. 02 (2017) 033, Note that the fifth force constraint shown in this paper cannot directly apply, since fifth force can happen within leptons or hadrons and if there is some cancellation between the leptonic and hadronic fifth forces, the constraint can easily be relaxed. Cancellation within only the leptonic sector with two mediators is also possible [26].

[26] C. Gross, O. Lebedev, and T. Toma, Cancellation Mechanism for Dark-Matter-Nucleon Interaction, Phys. Rev. Lett. 119, 191801 (2017).

[27] B. Batell, N. Lange, D. McKeen, M. Pospelov, and A. Ritz, Muon anomalous magnetic moment through the leptonic Higgs portal, Phys. Rev. D 95, 075003 (2017).

[28] S. Bergmann, Y. Grossman, and E. Nardi, Neutrino propagation in matter with general interactions, Phys. Rev. D 60 , 093008 (1999).

[29] J. Liao, D. Marfatia, and K. Whisnant, Generalized perturbations in neutrino mixing, Phys. Rev. D 92, 073004 (2015).

[30] An incomplete list: T. Ota, J. Sato, and N. a. Yamashita, Oscillation enhanced search for new interaction with neutrinos, Phys. Rev. D 65, 093015 (2002); A. Friedland, C. Lunardini, and M. Maltoni, Atmospheric neutrinos as probes of neutrino-matter interactions, Phys. Rev. D 70, 111301(R) (2004); S. Antusch, J. P. Baumann, and E. Fernandez-Martinez, Non-standard neutrino interactions with matter from physics beyond the standard model, Nucl. Phys. B810, 369 (2009).

[31] R. F. Sawyer, Do neutrinos have mass only within matter, Phys. Lett. B 448, 174 (1999).

[32] A. Gando et al. (KamLAND Collaboration), Constraints on $\theta_{13}$ from a three-flavor oscillation analysis of reactor antineutrinos at KamLAND, Phys. Rev. D 83, 052002 (2011).

[33] F. P. An et al. (Daya Bay Collaboration), Measurement of electron antineutrino oscillation based on 1230 days of operation of the Daya Bay experiment, Phys. Rev. D 95, 072006 (2017).

[34] S. H. Seo et al. (RENO Collaboration), Spectral measurement of the electron antineutrino oscillation amplitude and frequency using 500 live days of RENO data, Phys. Rev. D 98, 012002 (2018). 
[35] X. Qian, D. A. Dwyer, R. D. McKeown, P. Vogel, W. Wang, and C. Zhang, Mass hierarchy resolution in reactor antineutrino experiments: Parameter degeneracies and detector energy response, Phys. Rev. D 87, 033005 (2013); X. Qian, A. Tan, W. Wang, J. J. Ling, R. D. McKeown, and C. Zhang, Statistical evaluation of experimental determinations of neutrino mass hierarchy, Phys. Rev. D 86, 113011 (2012); S. F. Ge, K. Hagiwara, N. Okamura, and Y. Takaesu, Determination of mass hierarchy with medium baseline reactor neutrino experiments, J. High Energy Phys. 05 (2013) 131; F. An et al. (JUNO Collaboration), Neutrino Physics with JUNO, J. Phys. G 43, 030401 (2016); Z. Djurcic et al. (JUNO Collaboration), JUNO conceptual design report, arXiv:1508.07166.

[36] Y.F. Li, Y. Wang, and Z.z. Xing, Terrestrial matter effects on reactor antineutrino oscillations at JUNO or RENO-50: How small is small, Chin. Phys. C 40, 091001 (2016).

[37] S.-F. Ge, NuPro: A simulation package for neutrino properties, http://nupro.hepforge.org/.

[38] N. Vinyoles, A. M. Serenelli, F. L. Villante, S. Basu, J. Bergström, M. C. Gonzalez-Garcia, M. Maltoni, C. PeñaGaray, and N. Song, A new generation of standard solar models, Astrophys. J. 835, 202 (2017).

[39] B. Aharmim et al. (SNO Collaboration), combined analysis of all three phases of solar neutrino data from the sudbury neutrino observatory, Phys. Rev. C 88, 025501 (2013).

[40] M. Maltoni and A. Y. Smirnov, Solar neutrinos and neutrino physics, Eur. Phys. J. A 52, 87 (2016).

[41] M. Agostini et al. (Borexino Collaboration), First simultaneous precision spectroscopy of $p p,{ }^{7} \mathrm{Be}$, and pep solar neutrinos with borexino phase-II, arXiv:1707.09279.

[42] G. Bellini et al. (Borexino Collaboration), Measurement of the solar 8B neutrino rate with a liquid scintillator target and $3 \mathrm{MeV}$ energy threshold in the Borexino detector, Phys. Rev. D 82, 033006 (2010).

[43] Z. Djurcic et al. (JUNO Collaboration), JUNO conceptual design report, arXiv:1508.07166.

[44] J. F. Beacom et al. (Jinping Collaboration), Physics prospects of the Jinping neutrino experiment, Chin. Phys. C 41, 023002 (2017).

[45] S. F. Ge, K. Hagiwara, and C. Rott, A novel approach to study atmospheric neutrino oscillation, J. High Energy Phys. 06 (2014) 150.
[46] M. G. Aartsen et al. (IceCube PINGU Collaboration), Letter of intent: The precision IceCube next generation upgrade (PINGU), arXiv:1401.2046.

[47] S. Adrian-Martinez et al. (KM3Net Collaboration), Letter of intent for KM3NeT 2.0, J. Phys. G 43, 084001 (2016).

[48] M. S. Athar et al. (INO Collaboration), India-based neutrino observatory: Project Report, Vol. I, INO-2006-01 [inSpire].

[49] S. Razzaque and A. Y. Smirnov, Super-PINGU for measurement of the leptonic $C P$-phase with atmospheric neutrinos, J. High Energy Phys. 05 (2015) 139.

[50] J. Evslin, S. F. Ge, and K. Hagiwara, The leptonic $C P$ phase from $\mathrm{T} 2(\mathrm{H}) \mathrm{K}$ and $\mu^{+}$decay at rest, J. High Energy Phys. 02 (2016) 137; S. F. Ge, Measuring the leptonic dirac CP phase with TNT2K, arXiv:1704.08518.

[51] C. Adams et al. (LBNE Collaboration), The long-baseline neutrino experiment: Exploring fundamental symmetries of the universe, arXiv:1307.7335; R. Acciarri et al. (DUNE Collaboration), Long-baseline neutrino facility (LBNF) and deep underground neutrino experiment (DUNE): Conceptual design report, Vol. 2, The physics program for DUNE at LBNF, arXiv:1512.06148; R. Acciarri et al. (DUNE Collaboration), Long-baseline neutrino facility (LBNF) and deep underground neutrino experiment (DUNE): Conceptual design report, Vol. 1, The LBNF and DUNE projects, arXiv:1601.05471.

[52] O. G. Miranda, M. Tortola, and J. W. F. Valle, New Ambiguity in Probing CP Violation in Neutrino Oscillations, Phys. Rev. Lett. 117, 061804 (2016); S. F. Ge, P. Pasquini, M. Tortola, and J.W. F. Valle, Measuring the leptonic $C P$ phase in neutrino oscillations with nonunitary mixing, Phys. Rev. D 95, 033005 (2017).

[53] Y. Itow et al. (T2K Collaboration), The JHF-Kamioka neutrino project, arXiv:hep-ex/0106019; K. Abe et al., Letter of intent: The hyper-gamiokande experimentDetector design and physics potential, arXiv:1109.3262; K. Abe et al. (Hyper-Kamiokande Working Group), A long baseline neutrino oscillation experiment using J-PARC neutrino beam and hyper-kamiokande, arXiv:1412.4673; K. Abe et al. (Hyper-Kamiokande Proto- Collaboration), Physics potential of a long-baseline neutrino oscillation experiment using a J-PARC neutrino beam and hyperkamiokande, Prog. Theor. Exp. Phys. (2015), 053C02.

[54] D. S. Ayres et al. (NOvA Collaboration), NOvA: Proposal to build a 30 kiloton off-axis detector to study $\nu_{\mu} \rightarrow \nu_{e}$ oscillations in the NuMI beamline, arXiv:hep-ex/0503053. 